\title{
Manifestaciones clínicas inusuales del dengue hemorrágico en niños
}

\author{
Ángela Méndez, Gerardo González \\ Departamento de Pediatría, Universidad Industrial de Santander, Bucaramanga, Colombia.
}

Introducción. Recientemente han venido aumentando los reportes de dengue hemorrágico con manifestaciones inusuales, principalmente neurológicas y hepáticas, que aumentan la morbimortalidad.

Objetivo. Describir las manifestaciones inusuales del dengue hemorrágico en niños. Materiales y métodos. En el Departamento de Pediatría del Hospital Universitario de Santander en Bucaramanga, Colombia, de 913 pacientes con diagnóstico de dengue hemorrágico entre 1992 y 2004, se seleccionaron aquéllos que presentaron manifestaciones inusuales. Se clasificaron de acuerdo con los criterios de definición de caso, se agruparon según los órganos o sistemas principalmente comprometidos y se describieron los principales hallazgos clínicos, de laboratorio y relativos a la evolución.

Resultados. Se encontraron 168 pacientes que presentaron manifestaciones inusuales, con predominio del sexo masculino, edad escolar y grados III y IV de dengue hemorrágico. Las manifestaciones más frecuentes fueron hepáticas, $53(27 \%)$, y neurológicas, $49(25 \%)$, además de renales, 14 (7\%); cardíacas, 15 (8\%); pulmonares, 18 (9\%); colecistitis alitiásica, $18(9 \%)$; síndrome hemofagocítico, 5 (2,5\%); pancreatitis, 2 (1\%), y 21 casos de abdomen agudo (11\%). Fallecieron 10 pacientes.

Conclusión. Se analizan los resultados y se comparan con otros de países endemoepidémicos de dengue; se discute el significado e importancia de las manifestaciones clínicas inusuales, su difícil diagnóstico, casi siempre en forma retrospectiva, y se destaca la frecuencia de las manifestaciones hepáticas y neurológicas que pueden aumentar la morbimortalidad, ante las cuales deben estar alertas los médicos que trabajan en zonas endémicas para reconocerlas.

Palabras clave: dengue/complicaciones, fiebre por dengue hemorrágico/diagnóstico, hepatitis/ etiología, encefalitis/diagnóstico, niño.

\section{Abnormal clinical manifestations of dengue hemorrhagic fever in children}

Introduction. Recently, dengue hemorrhagic fever with unusual neurological and hepatic manifestations has been reported in children, with a concomitant increase in morbidity and mortality.

Objective. To describe unusual clinical manifestations of dengue hemorrhagic fever in children. Materials and methods. These manifestations were systematically scrutinized in dengue cases occurring between 1992-2004 in the Pediatrics Department of the University Hospital of Santander, Bucaramanga, Colombia. Case reports were examined of 913 patients with clinical symptoms of dengue hemorrhagic fever. They were classified according to standard criteria for dengue hemorrhagic fever and grouped according to the main organs or systems that were affected. Clinical presentation, laboratory results and medical evolution were summarized.

Results. Of the 913 cases, 168 showed abnormal clinical manifestations; these were predominant in male school age children and associated mainly with grades III and IV of dengue hemorrhagic fever. The most frequent manifestations were as follows: hepatitis- 53 cases $(27 \%)$, neurological alterations-49 (25\%), renal impairment-14 (7\%), cardiac involvement-15 (8\%), pulmonary alterations-18 (9\%), alithiasic cholecistitis-18 (9\%), haemophagocitic syndrome-5 (2.5\%), pancreatitis-2 (1\%), and acute abdominal pain-21 (11\%). Ten patients died.

Conclusions. The results were compared with data from other endemoepidemic countries of dengue. The significance and importance of abnormal dengue were explored, as well as the 
difficult retrospective diagnoses. Hepatic and neurological manifestations were recognized as frequently involved in elevated morbidity and mortality.Physicians working in endemic zones should be aware of them in order to recognize them.

Key Words: dengue/complications, dengue hemorrhagic fever/diagnosis, encephalitis/ diagnosis, hepatitis/etiology, child.

Con el reconocimiento de las formas complicadas del dengue, el dengue hemorrágico y el síndrome de choque por dengue, la enfermedad ha recibido gran atención mundial y hoy día es considerada la enfermedad hemorrágica viral más importante y la virosis transmitida por artrópodos más ampliamente diseminada en los países tropicales y subtropicales (1). Colombia es uno de los países en los cuales está aumentando alarmantemente su incidencia, y en gran parte de su territorio existe la enfermedad o se está en riesgo. La gravedad de la enfermedad está determinada principalmente por la fuga plasmática debido al aumento de la permeabilidad vascular y por las alteraciones en la hemostasis. Se pensaba que el compromiso de órganos vitales era secundario al choque o a las hemorragias, pero desde hace tres décadas se han venido reportando casos de infección por dengue con manifestaciones que no pueden asociarse a otros factores o condiciones diferentes, y se han presentado manifestaciones inusuales de la enfermedad, entre las cuales se destacan las neurológicas y las hepáticas, las cuales aumentan la morbimortalidad (2-5). El objetivo de este estudio fue describir las manifestaciones inusuales del dengue hemorrágico en niños.

\section{Materiales y métodos}

Este es un estudio descriptivo de casos realizado en el Hospital Universitario de Santander (HUS) (anteriormente Hospital Universitario Ramón González Valencia), entidad del Estado, de tercer nivel y centro de referencia localizado en Bucaramanga, ciudad del nororiente de Colombia,

\footnotetext{
$\overline{\text { Correspondencia: }}$

Ángela Méndez

Calle 45 bis № 27-75 apto 301, Torres de Sotomayor,

Bucaramanga, Colombia

Teléfonos: 577 6470123; fax: 5776786048

amendezb@telecom.com.co; gergonve@yahoo.com
}

Recibido: 24/05/05; aceptado: 23/10/05 a una altitud de $1000 \mathrm{~m}$, clima tropical con temperatura promedio de $22^{\circ} \mathrm{C}$ y, aproximadamente, 1'000.000 de habitantes en su área metropolitana (cuatro municipios circunvecinos con facilidades de acceso y a menos de 30 minutos de distancia). El Departamento de Pediatría admite pacientes referidos o de consulta de urgencias hasta los 12 años de edad y son atendidos por pediatras especialistas, profesores de la universidad y médicos residentes. Se clasifican por grupos etarios así: recién nacidos (hasta 30 días), lactantes (31 días a 24 meses), pre-escolares (2 a 5 años) y escolares (6 a 12 años).

Desde 1992, los autores vienen haciendo seguimiento de los pacientes que han sido hospitalizados con diagnóstico de dengue hemorrágico por medio de un formulario con los principales datos sociodemográficos, clínicos y paraclínicos, información que luego se consigna en una base de datos de Epi-info 6.04. Los primeros resultados obtenidos después de 10 años de seguimiento ya fueron publicados (6). Para el presente estudio se realizó una revisión retrospectiva de la base de datos desde enero de 1992 hasta diciembre de 2004. Se revisaron e incluyeron las historias clínicas de aquellos pacientes con diagnóstico probable de dengue hemorrágico, cuyas manifestaciones se consideraron diferentes a las contempladas en la definición de casos para dengue y dengue hemorrágico de la Organización Mundial de la Salud (OMS) (7) que no pudieron explicarse por una enfermedad concomitante, y en quienes no se encontraron factores o condiciones que explicaran las manifestaciones inusuales. Se excluyeron las historias clínicas de pacientes que no tuvieron confirmación inmunológica mediante pruebas pareadas de inhibición de hemaglutinación en 1992, y desde 1993 por determinación de lgM específica para dengue mediante prueba inmunoenzimática de captura (UMELISA, Centro 
de Inmunoensayo, La Habana, Cuba), realizada por el Laboratorio Departamental de la Secretaría de Salud de Santander en una muestra de suero de convaleciente y reportada al HUS. La clasificación por grados de gravedad de dengue hemorrágico se hizo según los criterios de la OMS (7). Según los órganos o sistemas principalmente comprometidos, las manifestaciones se clasificaron en hepáticas, neurológicas, colecistitis, pancreatitis, renales, cardíacas, pulmonares, síndrome hemofagocítico y un grupo que presentó abdomen agudo.

\section{Resultados}

En el periodo estudiado (1992 a 2004), 913 pacientes egresaron con diagnóstico de dengue hemorrágico, lo cual representa una frecuencia relativa de $1,5 \%$ del total de egresos pediátricos, y entre éstos se encontraron 168 pacientes $(18,5 \%)$ en quienes se identificaron una o más manifestaciones inusuales. En el cuadro 1 se resume el número de cada una de las manifestaciones inusuales (195 en total) y se indica el género, el grupo etario y el grado de gravedad de dengue hemorrágico. Once de las historias que no pudieron clasificarse en ningún grado de dengue hemorrágico correspondieron a pacientes en quienes los exámenes practicados no demostraron trombocitopenia de 100.000 o menos, pero sí hemorragias y signos de fuga vascular; un paciente que presentó hepatitis, insuficiencia hepática y encefalopatía sólo reunía los criterios para ser clasificado como dengue clásico. Las 195 manifestaciones inusuales encontradas se resumen en el cuadro 2, en el que se registra que 47 pacientes presentaron una sola manifestación inusual y los demás dos o más manifestaciones inusuales asociadas, observándose que las más frecuentes fueron las hepáticas y las neurológicas.

Cuadro 1. Manifestaciones inusuales en pacientes con dengue hemorrágico.

\begin{tabular}{|c|c|c|c|c|c|c|c|c|c|c|c|c|}
\hline \multirow{2}{*}{$\begin{array}{l}\text { Manifestaciones } \\
\text { inusuales }\end{array}$} & \multirow[t]{2}{*}{ Total } & \multicolumn{2}{|c|}{ Sexo } & \multicolumn{4}{|c|}{ Grupo etario } & \multicolumn{5}{|c|}{ Grado de gravedad } \\
\hline & & $\mathbf{M}$ & $\mathbf{F}$ & $\mathbf{R N}$ & $\mathbf{L}$ & PE & $\bar{E}$ & I & II & III & IV & NC \\
\hline Hepáticas & $53(27 \%)$ & 28 & 25 & 1 & 14 & 14 & 24 & 1 & 20 & 11 & 16 & 5 \\
\hline Neurológicas & $49(25 \%)$ & 26 & 23 & 1 & 15 & 13 & 20 & & 7 & 12 & 28 & 2 \\
\hline Colecistitis & $18(9 \%)$ & 14 & 4 & & 2 & 7 & 9 & & 7 & 5 & 6 & \\
\hline Pancreatitis & $2(1 \%)$ & 1 & 1 & & & 1 & 1 & & & 1 & 1 & \\
\hline Renales & $14(7 \%)$ & 13 & 1 & 1 & 1 & 6 & 6 & & & 6 & 8 & \\
\hline Cardíacas & $15(8 \%)$ & 8 & 7 & & 1 & 6 & 8 & & 5 & 4 & 6 & \\
\hline Pulmonares & $18(9 \%)$ & 9 & 9 & 1 & 3 & 7 & 7 & & 1 & 5 & 12 & \\
\hline Síndrome hemofagocítico & $5(2,5 \%)$ & 2 & 3 & & 1 & 1 & 3 & & 3 & 1 & 1 & \\
\hline Abdomen agudo & $21(11 \%)$ & 11 & 10 & & 1 & 2 & 18 & & 6 & 5 & 6 & 4 \\
\hline
\end{tabular}

RN: recién nacidos; L: lactantes; PE: preescolar; E: escolar; NC: sin clasificación.

Cuadro 2. Asociación de manifestaciones inusuales en dengue hemorrágico.

\begin{tabular}{|c|c|c|c|c|c|c|c|c|c|c|}
\hline Manifestaciones inusuales & Hep & $\mathrm{Neu}$ & Col & Pan & Ren & Car & Pul & Shf & Abd & Total \\
\hline Hepáticas (Hep) & 3 & 22 & 9 & 2 & 3 & 4 & 6 & & 4 & 53 \\
\hline Neurológicas (Neu) & 22 & 13 & & & 10 & 2 & 2 & & & 49 \\
\hline Colecistitis (Col) & 9 & & & & & 1 & & 2 & 6 & 18 \\
\hline Pancreatitis (Pan) & 2 & & & & & & & & & 2 \\
\hline Renales (Ren) & 3 & 10 & & & & & 1 & & & 14 \\
\hline Cardiacas (Car) & 4 & 2 & 1 & & & 8 & & & & 15 \\
\hline Pulmonares (Pul) & 6 & 2 & & & 1 & & 9 & & & 18 \\
\hline Síndrome hemofagocítico (Shf) & & & 2 & & & & & 3 & & 5 \\
\hline Abdomen agudo (Abd) & 4 & & 6 & & & & & & 11 & 21 \\
\hline Total & 53 & 49 & 18 & 2 & 14 & 15 & 18 & 5 & 21 & 195 \\
\hline
\end{tabular}


Cuadro 3. Parámetros de laboratorio clínico en pacientes con manifestaciones hepáticas.

\begin{tabular}{lccc}
\hline Prueba & $\mathbf{n = 5 3}$ & Rango & Mediana \\
\hline Aspartato aminotransferasa (AST)(UI) & $53 / 53$ & $100-7117$ & 484 \\
Alanino aminotransferasa (ALT)(UI) & $53 / 53$ & $100-1927$ & 400 \\
Bilirrubina directa (mg/dl) & $9 / 53$ & $2-16,5$ & 6,43 \\
Tiempo de protrombina (s) & $48 / 53$ & $12-50$ & 18,6 \\
Tiempo parcial de tromboplastina (s) & $48 / 53$ & $55-245$ & 64,3 \\
Albúmina (g/dl) & $50 / 53$ & $1,5-3,5$ & 2,53 \\
\hline
\end{tabular}

\section{Manifestaciones hepáticas}

Presentaron compromiso hepático 53 pacientes, cuyos parámetros de laboratorio se resumen en el cuadro 3. Se realizaron pruebas de bilirrubina solamente en los pacientes que clínicamente presentaron ictericia. El tiempo de protrombina y el parcial de tromboplastina se encontraron prolongados en 23 de 48 . Se hicieron pruebas serológicas para diagnóstico diferencial con otras hepatitis virales en 10 pacientes, con resultados negativos. Hubo complicaciones por hemorragia del tracto digestivo alto en 22 pacientes $(41,5 \%)$, por encefalopatía en $22(41,5 \%)$, y por insuficiencia hepática fulminante y coagulación intravascular diseminada (CID) en tres (5,7\%). En todos los casos, el tratamiento fue de soporte; 47 pacientes se recuperaron y 6 fallecieron.

\section{Manifestaciones neurológicas}

Se clasificaron 46 pacientes con encefalopatía y tres con encefalitis. En el cuadro 4 se resumen las manifestaciones clínicas observadas en este grupo. La encefalopatía se consideró cuando se identificaron causas secundarias, entre las cuales 22 fueron hepatitis; 10, insuficiencia renal aguda, 10 , hipoperfusión por choque y 4 , desequilibrio hidroelectrolítico causado por hiponatremia. Se realizó examen de líquido cefalorraquídeo (LCR) en 25 pacientes con resultados normales. Se realizó tomografía cerebral (TC) a 20 , de los cuales 11 mostraron edema cerebral difuso y uno, edema focalizado e infartos isquémicos. En cuanto a la evolución, 40 mejoraron al final de la primera semana de enfermedad, dos tardaron más tiempo en recuperarse y necesitaron rehabilitación (uno con infarto cerebral isquémico y el otro con estado convulsivo), y cuatro fallecieron en choque.
Cuadro 4. Manifestaciones neurológicas en pacientes con dengue hemorrágico.

\begin{tabular}{lrrrr}
\hline Manifestaciones & $\begin{array}{c}\text { Encefalopatía } \\
(\mathbf{n = 4 6 )}\end{array}$ & \multicolumn{2}{c}{$\begin{array}{c}\text { Encefalitis } \\
(\mathbf{n}=\mathbf{3})\end{array}$} \\
\hline Cefalea & 22 & $(55 \%)$ & 3 & $(10 \%)$ \\
Vómito & 46 & $(100 \%)$ & 3 & $(100 \%)$ \\
Convulsiones & 42 & $(93 \%)$ & 3 & $(100 \%)$ \\
Trastornos de conciencia & 38 & $(84 \%)$ & 3 & $(100 \%)$ \\
Trastornos de conducta & 18 & $(40 \%)$ & 3 & $(100 \%)$ \\
Focalización & & & 1 & $(33 \%)$ \\
Signos meníngeos & & & 2 & $(67 \%)$ \\
Mortalidad & & $4 / 46$ & & $2 / 3$ \\
\hline
\end{tabular}

Tres pacientes fueron clasificados en el grupo de encefalitis porque no fue posible explicar su complicación por otra causa, pero no se logró demostración etiológica. En el examen de LCR, dos pacientes mostraron pleocitosis a expensas de los linfocitos y uno fue normal. La TC mostró edema cerebral difuso en estos pacientes, en uno de ellos se informó encefalitis aguda diseminada y en otro, infarto isquémico frontooccipital. La resonancia magnética se hizo en un paciente, cuyos resultados revelaron lesiones inflamatorias en el hemisferio cerebral izquierdo. Dos pacientes fallecieron y el otro requirió rehabilitación al persistir la hemiparesia, pero el resultado final fue favorable.

\section{Colecistitis alitiásica}

Ésta se observó en 18 casos, en 17 de los cuales se realizó ecografía hepatobiliar, y se observó vesícula con edema de paredes, distendida, alitiásica y ascitis; a un paciente que presentó signos de abdomen agudo se le hizo el diagnóstico por observación directa durante laparotomía. La evolución final fue buena con manejo de soporte, excepto en un paciente que se complicó con choque durante la cirugía y murió en el postoperatorio. 


\section{Pancreatitis}

Ésta se diagnosticó en dos pacientes. Se encontraron amilasas y fosfatasa alcalina elevadas, glucemia normal e hipocalcemia en un paciente; la ecografía abdominal informó páncreas edematoso en ambos. Los dos evolucionaron bien con manejo de soporte.

\section{Manifestaciones renales}

Catorce pacientes presentaron insuficiencia renal aguda, en dos de ellos se diagnosticó síndrome hemolítico urémico y en uno glomerulonefritis aguda. Tres requirieron diálisis peritoneal, 11 evolucionaron bien y los tres que fallecieron presentaban más de dos manifestaciones inusuales.

\section{Manifestaciones cardíacas}

Se observó derrame pericárdico en siete pacientes que tuvieron más de un derrame seroso; en todos se confirmó por ecocardiograma y la evolución fue buena sin tratamiento específico. Se diagnosticó miocarditis en cuatro pacientes, uno asociado a derrame pericárdico y otro a hepatitis con encefalopatía. La radiografía de tórax mostraba cardiomegalia, el electrocardiograma y el ecocardiograma cambios compatibles con miocarditis, y en uno el diagnóstico se hizo en la autopsia; dos evolucionaron bien y dos fallecieron. Cuatro pacientes presentaron bradicardia sinusal durante la convalecencia y la evolución fue buena sin tratamiento específico.

\section{Manifestaciones pulmonares}

En la fase afebril, durante la administración de líquidos parenterales, 13 pacientes presentaron edema pulmonar; todos cursaron con edemas periféricos, requirieron manejo con furosemida y evolucionaron bien, excepto un paciente que falleció y cuya autopsia reveló, además, neumonitis intersticial y miocarditis. Se presentó síndrome de disnea en dos pacientes que fueron manejados en cuidado intensivo, pues requirieron ventilación mecánica; uno de ellos se complicó con neumonía nosocomial. Se presentó hemorragia pulmonar masiva en tres pacientes con dengue hemorrágico grado IV y todos fallecieron.

\section{Síndrome hemofagocítico}

Éste se encontró en cinco pacientes con dengue hemorrágico que cursaron con fiebre prolongada y citopenias persistentes. En el aspirado de medula ósea se encontraron histiocitos fagocitando células hemáticas de la serie eritroide, mieloide o plaquetaria. La evolución fue buena a partir de la tercera semana de enfermedad.

\section{Abdomen agudo}

De los 21 pacientes clasificados en el grupo de abdomen agudo, a cuatro se les practicó laparotomía exploradora, y se observó ascitis gelatinosa. Un paciente que presentaba colecistitis falleció en el postoperatorio.

\section{Mortalidad}

De los pacientes estudiados fallecieron 10 , todos con más de una manifestación inusual. Se practicó autopsia a cuatro pacientes, y se encontró en todos derrames serosos, microhemorragias en vísceras y edema cerebral. Un paciente presentó herniación de amígdalas cerebelosas y meningoencefalitis aguda; tres, hepatitis con cambios grasos y necrosis focal de hepatocitos; dos, necrosis tubular aguda, uno con fibrina en capilares glomerulares; dos, miocarditis; uno, hemorragia subendocárdica; uno, hemorragia intersticial en septum; uno, neumonitis intersticial y uno, edema pulmonar.

\section{Discusión}

Las manifestaciones inusuales del dengue se han descrito desde hace aproximadamente tres décadas $y$, desde entonces se han reportado numerosos pacientes con compromiso hepático y neurológico principalmente, con una mortalidad alta comparada con la de casos de dengue hemorrágico/síndrome de choque por dengue $(4,5)$. Antes de concluir que los hallazgos atípicos son manifestaciones inusuales del dengue, debería buscarse si éstas se acompañan de infección dual o coinfección, ya que pueden pasarse por alto en el diagnóstico o retardarlo, afectando así el tratamiento del dengue debido a que modifican la presentación clínica (8).

En esta revisión encontramos dengue hemorrágico con una frecuencia relativa de 1,5\% 
en relación al total de egresos en el período estudiado (3\% en el año 2001). La proporción de manifestaciones inusuales (18,5\%) se informa sólo con respecto a dengue hemorrágico por cuanto los pacientes con dengue clásico normalmente no son hospitalizados. La frecuencia con respecto a género y edad conservó las mismas proporciones que habíamos reportado para toda la serie de casos de dengue hemorrágico, destacándose la mayor frecuencia en la edad escolar (6).

En los pacientes del estudio sólo se reportó un aislamiento viral Den-2 en una paciente clasificada como dengue hemorrágico y hepatitis por dengue. En relación a los serotipos circulantes durante estos años en la región, Den-1 y Den-2 habían circulado hasta 1999, en el 2000 se aisló Den-4 y en agosto de 2001 se aisló Den-3, el cual fue predominante en 2003 y 2004 en el área metropolitana de Bucaramanga (9).

En nuestro estudio, de los 53 pacientes que cursaron con compromiso hepático, sólo en 9 se observó ictericia e hiperbilirrubinemia a expensas de la bilirrubina directa. El diagnóstico se hizo con base en las aminotransferasas elevadas, tal como se ha informado en otros estudios (10). Las manifestaciones hepáticas se describen tanto en dengue clásico como en dengue hemorrágico, ya sea en formas primarias o secundarias, y la lesión hepática se superpone al cuadro clínico de esta entidad, que puede generar una hepatitis anictérica $(11,12)$. En los estudios sobre la alteración de la función hepática en dengue se ha encontrado que la AST se eleva más que la ALT, diferencia rara vez vista en la hepatitis viral (13). En la presentación de la falla hepática aguda pueden intervenir varios factores como son la invasión directa del virus y su replicación en el hígado, la hipoperfusión secundaria a choque prolongado, a medicamentos hepatotóxicos o al daño hepático previo (4). El curso de la enfermedad es autolimitado, pero puede complicarse con hepatitis fulminante y falla hepática, la cual es una de las causas más importantes de encefalopatía y muerte (14).

El compromiso neurológico en infecciones por dengue se consideraba poco frecuente, y la asociación con manifestaciones neurológicas inusuales fue reportada por primera vez en 1976 en Tailandia (2) como "encefalopatía aguda asociada con infección por dengue"; desde entonces, la incidencia ha ido en aumento y diversos autores han reportado casos similares en varios países del sudeste asiático, posteriormente en islas del Pacífico, en Australia, en la India y, más recientemente, en América $(5,15-20)$. En 1996, Lum y colaboradores presentaron un estudio en 6 pacientes pediátricos, en quienes, por medio de técnicas diagnósticas más nuevas, encontraron evidencia sólida de que el virus dengue cruza la barrera hematoencefálica e invade directamente el cerebro causando encefalitis en infecciones tanto primarias como secundarias, y en las formas leves y complicadas (21). Actualmente se reconoce que las manifestaciones del sistema nervioso central (SNC) en infecciones por dengue pueden ocurrir en cualquier estadio, con cualquier grado de gravedad y por acción de cualquier serotipo, asociándose más frecuente-mente con Den 2 y Den 3 (21-24).

La encefalopatía puede explicarse por efectos indirectos de infecciones dengue en el SNC, y se atribuye a varios factores como hipoperfusión cerebral por hipotensión (fuga vascular), y sus complicaciones electrolíticas y metabólicas como la hiponatremia y la hipoglucemia, la hipoxia cerebral, el edema cerebral o la encefalopatía hepática en pacientes con compromiso hepático grave o falla hepática fulminante, lo cual puede ser parte de un síndrome semejante al de Reye; la hemorragia cerebral (subaracnoidea); la hemorragia microcapilar y la liberación de sustancias tóxicas $(4,20-25)$.

Pancharoen y Thisyakorn definieron como encefalíticos a aquellos pacientes que tuvieron alteración de conciencia de tipo estupor, coma y confusión mental, sin que se reconociera en ellos infección por dengue al comienzo de las manifestaciones neurológicas (26). En nuestros pacientes se hizo el diagnóstico en forma retrospectiva en la mayoría de los casos. El pronóstico usualmente es favorable, las propiedades neurovirulentas parecen ser benignas, autolimitadas y la mayoría de los pacientes se recupera al final de la primera 
semana de enfermedad sin acusar secuelas (27). En los pacientes con coma en el estudio de Solomon y colaboradores, el tiempo de recuperación promedio fue de 3 a 5 días (23). La evolución de nuestros pacientes fue similar. Las manifestaciones clínicas de comienzo agudo, como fiebre y alteración del nivel de conciencia o confusión mental, son indistinguibles de cualquier otro tipo de encefalitis viral; por tanto, en áreas endémicas de infecciones por dengue, y en pacientes con manifestaciones neurológicas debería considerarse éste como otra causa posible de encefalopatía o encefalitis (28).

También se han descrito, principalmente en adultos y tardíamente, manifestaciones del sistema nervioso periférico como polineuritis aguda, polirradiculoneuritis o síndrome de GuillainBarré (29), pero éstas no se observaron en nuestra serie.

El compromiso de la vesícula es un hallazgo poco frecuente que se considera como un signo pronóstico de enfermedad grave e inminencia de choque. En el estudio realizado en Taiwan por Wu y colaboradores se reportó en $8 \%$ de los pacientes con dengue hemorrágico (30); otros informes han documentado engrosamiento de la pared de la vesícula biliar hasta en 18 a $28 \%$ de casos de dengue hemorrágico (31-33); Setiawan y colaboradores lo encontraron en $33 \%$ de los casos leves y en el $94 \%$ de los casos graves. Los hallazgos ecográficos deben correlacionarse con la clínica, debido a que un engrosamiento de las paredes no es específico para enfermedad primaria de la vesícula biliar (33). El curso de esta entidad es autolimitado, el tratamiento es sintomático y la colecistectomía no debería indicarse en estos pacientes, ya que la tendencia a presentar sangrados podría ser muy difícil de manejar durante y después de la cirugía.

La pancreatitis es una complicación rara del dengue hemorrágico. Setiawan y colaboradores estudiaron 142 niños con dengue o dengue hemorrágico y dolor abdominal, y encontraron agrandamiento del páncreas en $29 \%$ y $44 \%$ de los casos de dengue hemorrágico grados I-II y III-IV, respectivamente (34). Los dos pacientes de nuestro estudio evolucionaron satisfactoriamente con tratamiento de soporte.
La insuficiencia renal aguda en dengue hemorrágico y síndrome de choque por dengue se ha relacionado principalmente con la duración y la gravedad de la hipoperfusión renal cuando no se hace un adecuado reemplazo de líquidos durante la fase hipovolémica. El periodo de recuperación de la función renal es variable y puede prolongarse hasta por 4 a 6 semanas $(4,35)$. En Malasia se ha reportado síndrome hemolítico urémico y glomerulonefritis aguda por depósitos de inmunocomplejos (4). En nuestro estudio un paciente presentó síndrome hemolítico urémico y otro glomerulonefritis aguda postdengue.

Entre las manifestaciones cardíacas inusuales se observan, ocasionalmente, grandes colecciones pericárdicas que se reabsorben espontáneamente en 1 a 2 semanas, o pueden complicarse con miopericarditis. En nuestra serie se documentó en siete pacientes y todos tenían más de un derrame seroso. La miocarditis por virus dengue tiene una baja incidencia y pocas veces causa insuficiencia cardiaca; se han descrito cambios ecocardiográficos como dilatación ventricular izquierda, hipocinesia global e incompetencia mitral $(4,36)$. Los trastornos del ritmo cardíaco aparecen durante la infección por dengue y son atribuidos a la miocarditis viral. En el dengue hemorrágico y síndrome de choque por dengue puede observarse disfunción cardiaca aguda reversible, la cual podría ser responsable de la hipotensión y el choque; es necesario valorar la función miocárdica en pacientes con esta enfermedad, especialmente en aquéllos que tengan hipotensión persistente a pesar de una adecuada hidratación (4,36-38).

La mayoría de las complicaciones pulmonares se observaron después de la administración de líquidos endovenosos o transfusiones. Los pacientes con edema pulmonar mostraron, además, edemas periféricos, signos de hipervolemia y cursaron con presión arterial en percentiles superiores a los normales o elevados para su edad, y se consideraron como manifestación de hipervolemia en los casos en que se prolongó la terapia con líquidos endovenosos después de la fase de fuga vascular, por lo cual es necesaria una estrecha vigilancia 
clínica durante la administración de líquidos. También se ha descrito edema pulmonar en pacientes con hidrotórax bilateral, así como en periodos de choque, lo cual se correlaciona con la propia fuga vascular o puede presentarse por falla renal (4). El síndrome de disnea (o síndrome de disnea tipo adulto) es poco frecuente; se presenta cuando se administran rápidamente los cristaloides y se ha descrito más frecuentemente en niños (39). Thisyakorn encontró dos de 14 pacientes que tuvieron síndrome de choque por dengue y desarrollaron este síndrome durante la convalecencia de la enfermedad, y requirieron ventilación mecánica. Este síndrome y el edema pulmonar pueden complicarse con neumonía (5). Los pacientes de nuestra serie también presentaron este síndrome después de la fase de fuga vascular. En el curso del dengue hemorrágico puede observarse hemorragia pulmonar, manifestada por hemoptisis $(4,40)$. Los tres pacientes de nuestro estudio con esta complicación fallecieron. El síndrome hemofagocítico asociado al dengue se ha descrito ocasionalmente (41). Nosotros encontramos cinco pacientes que en el curso de la enfermedad tuvieron una evolución atípica, con fiebre prolongada como el signo más constante, lo cual obligó a realizar un aspirado de médula ósea como parte de la investigación del diagnóstico diferencial, encontrándose en todos ellos histiocitos fagocitando células hemáticas de la serie eritroide, mieloide o plaquetaria, sobre lo cual hicimos un primer reporte de tres casos. Esta entidad no requiere ningún tratamiento específico diferente a las medidas de soporte. Se recomienda que en niños con diagnóstico de dengue hemorrágico cuyo curso clínico sea atípico, con fiebre prolongada y citopenias persistentes, se debe practicar aspirado de médula ósea para descartar una enfermedad linfoproliferativa y por la posibilidad de que la causa de la complicación corresponda a un síndrome hemofagocítico secundario (42).

En esta revisión, entre los pacientes que presentaron dolor abdominal, 21 presentaron además signos de irritación peritoneal y se clasificaron como abdomen agudo; de estos, cuatro fueron intervenidos con laparotomía exploradora y se encontró ascitis gelatinosa, lo cual, asociado con trombocitopenia postoperatoria, hizo sospechar la presencia de dengue hemorrágico, hecho confirmado posteriormente. Estos pacientes evolucionaron satisfactoriamente con manejo médico, excepto un lactante que falleció en el postoperatorio de la laparotomía. EI dolor abdominal grave en el dengue se ha atribuido a varios factores como distensión de la cápsula hepática, estimulación de plexos nerviosos por la distensión del espacio retroperitoneal a causa del edema o por disminución de la perfusión mesentérica $(5,34)$, pero si se acompaña de signos de irritación peritoneal debe tenerse en cuenta en el diagnóstico diferencial del abdomen agudo en zonas endémicas de dengue para evitar laparotomías innecesarias.

La mortalidad por dengue hemorrágico en nuestro hospital se ha presentado principalmente en casos con manifestaciones inusuales, lo que se refleja en el hecho de que del total de 913 pacientes fallecieron 13, 10 de ellos con manifestaciones inusuales (6). Otros estudios también han reportado mayor mortalidad relacionada con manifestaciones inusuales (5). El diagnóstico se hace retrospectivamente en la mayoría de los casos, debido a que las manifestaciones clínicas son muy similares en otras enfermedades infecciosas y se pueden pasar por alto inicialmente, ya que no se tienen en cuenta en la definición de caso y no están consideradas en la actual clasificación de la OMS. De la revisión de los diferentes reportes sobre manifestaciones inusuales en el dengue, especialmente hepáticas y neurológicas, se concluye que se hace indispensable y urgente su inclusión entre los criterios de gravedad de la enfermedad. Por ahora es importante tenerlas en cuenta en el diagnóstico diferencial y los médicos que trabajan en zonas endemoepidémicas de dengue deben estar alertas para reconocerlas.

\section{Agradecimientos}

A José Gabriel Rigau-Pérez, pediatra epidemiólogo (CDC Dengue Branch, Puerto Rico) y a Ángela María Gutiérrez y Ángela María Pinzón, profesoras de la Universidad del Rosario, por la revisión y sugerencias en el manuscrito. 


\section{Conflicto de intereses}

Los autores declaran no tener ningún conflicto de intereses.

\section{Financiación}

Este trabajo no contó con ninguna fuente de financiación.

\section{Referencias}

1. Halstead SB. More dengue, more questions. Emerg Infect Dis 2005;11:740-1.

2. Sumarmo, Wulur H, Jahja E, Gubler DJ, Sutomenggold TS, Sulianti Saroso J. Encephalopathy associated with dengue Infection. Lancet 1978;1:449-50.

3. Alvarez ME, Ramírez-Ronda $\mathbf{C H}$. Dengue and hepatic failure. Am J Med 1985;79:670-4.

4. George R, Lum LC. Clinical spectrum of dengue infection. In: Gubler DJ, Kuno G, editores. Dengue and dengue hemorrhagic fever. Wallingford, Reino Unido: CAB International; 1997. p.89-113.

5. Thisyakorn $\mathbf{U}$, Thisyakorn C. Dengue infection with unusual manifestations. J Med Assoc Thai 1994;77:410-3.

6. Méndez A, González G. Dengue hemorrágico en niños: diez años de experiencia clínica. Biomédica 2003;23:180-93.

7. World Health Organization. Dengue haemorrhagic fever. Diagnosis, treatment, prevention and control. 2nd edition. Geneva: WHO; 1997. p.12-23

8. Pancharoen C, Thisyakorn U. Coinfections in dengue patients. Pediatr Infect Dis J 1998;17:81-2.

9. Ocazionez RE, Cortés F, Villar LA. Vigilancia del dengue basada en el laboratorio: diferencias en el número de casos y virus aislados según la recolección del suero y la prueba serológica. Colomb Med 2005;36:65-72

10. Mohan B, Patwari AK, Anand VK. Hepatic dysfunction in childhood dengue infection. J Trop Pediatr 2000;46:40-3.

11. del Valle S, Piñera M, Guash F. Hepatitis reactiva por virus del dengue hemorrágico. Rev Cubana Med Trop 2001;53:28-31.

12. de Souza LJ, Goncalves Carneiro H, Souto Filho JT, Ferreira de Souza T, Azevedo Cortes V, Neto CG et al. Hepatitis in dengue shock syndrome. Braz J Infect Dis 2002;6:322-7.

13. Nguyen TL, Nguyen TH, Tieu NT. The impact of dengue haemorrhagic fever on liver function. Res Virol 1977;148:273-7.
14. Lawn SD, Tilley R, Lloyd G, Finlayson C, Tolley H, Newman $\mathbf{P}$ et al. Dengue hemorrhagic fever with fulminant hepatic failure in an inmigrant returning to Bangladesh. Clin Infect Dis 2003;37:e1-4.

15. Cabrera JA, Lopes OJ, Hernández E. Alteraciones electroencefalográficas en la fiebre hemorrágica de dengue. Revisión sobre sus manifestaciones neurológicas. Rev Cub Med 1984;23:468-78.

16. Jiménez DR, Santana JL, Ramírez-Ronda $\mathbf{C H}$. Neurological disorders associated to dengue infection. Bol Asoc Med PR 1988;80:208-11.

17. Ferreira ML, Cavalcanti CG, Coelho CA, Mesquita SD. Manifestações neurológicas de dengue: estudo de 41 casos. Arq Neuropsiquiatr 2005;63:488-93.

18. Rajajee S, Mukundan D. Neurological manifestations in dengue hemorrhagic fever. Indian Pediatr 1994;31:688-90.

19. Row D, Weinstein P, Murray-Smith S. Dengue fever with encephalopathy in Australia. Am J Trop Med Hyg 1996;54:253-5.

20. Palma-da Cunha-Matta A, Soares-Moreno SA, Cardosode Almeida A, Aquilera-de Freitas V, Carod-Artal FJ. Complicaciones neurológicas de la infección por el virus del dengue. Rev Neurol 2004;39:233-7.

21. Lum LC, Lam SK, Choy YS, George R, Harun F. Dengue encephalitis: a true entity? Am J Trop Med Hyg 1996;54:256-9.

22. Cam BV, Fonsmark L, Hue NB, Phuong NT, Poulsen A, Heegaard ED. Prospective case-control study of encephalopathy in children with dengue hemorrhagic fever. Am J Trop Med Hyg 2001;65:848-51.

23. Solomon T, Dung NM, Vaughn DW, Kneen R, Thao LT, Raengsakulrach B et al. Neurological manifestations of dengue infection. Lancet 2000;355:1053-9.

24. Vasconcelos PF, da Rosa AP, Coelho IC, Menezes DB, da Rosa ES, Rodrigues SG et al. Involvement of the central nervous system in dengue fever: three serologically confirmed cases from Fortaleza Ceara, Brazil. Rev Inst Med Trop Sao Paulo 1998;40:35-9.

25. Chimelli L, Hahn MD, Netto MB, Ramos RG, Dias M, Gray F. Dengue: neuropathological findings in 5 fatal cases from Brazil. Clin Neuropathol 1990;9:157-62.

26. Pancharoen C, Thisyakorn U. Neurological manifestations in dengue patients. Southeast Asian J Trop Med Public Health 2001;32:341-5.

27. Kankirawatana $\mathbf{P}$, Chokephaibulkit $\mathbf{K}$, Puthavathana $\mathbf{P}$, Yoksan S, Apintanapong S, Pongthapisit V. Dengue infection presenting with central nervous system manifestation. J Child Neurol 2000;15:544-7.

28. Garcia-Rivera EJ, Rigau-Perez JG. Encephalitis and dengue. Lancet 2002;360:261.

29. Santos NQ, Azoubel AC, Lopes AA, Costa G, Bacellar A. Guillain-Barre syndrome in the course of dengue: case report. Arq Neuropsiquiatr 2004;62:144-6. 
30. Wu KL, Changchien CS, Kuo CM, Chuah SK, Lu SN, Eng HL et al. Dengue fever with acute acalculous cholecystitis. Am J Trop Med Hyg 2003;68:657-60.

31. Pramuljo HS, Harun SR. Ultrasound findings in dengue haemorrhagic fever. Pediatr Radiol 1991;21:100-2.

32. Reynes JM, Laurent A, Deubel V, Telliam E, Moreau JP. The first epidemic of dengue hemorrhagic fever in French Guiana. Am J Trop Med Hyg 1994;51:545-53.

33. Setiawan MW. Samsi TK, Wulur H, Sugianto D, Pool TN. Dengue haemorrhagic fever: ultrasound as an aid to predict the severity of the disease. Pediatr Radiol 1998;28:1-4.

34. Setiawan MW, Samsi TK, Wulur H, Sugianto D, Pool TN. Epigatric pain and sonographic assessment of the pancreas in dengue hemorrhagic fever. J Clin Ultrasound 1998;26:257-9.

35. Hommel D, Talarmin A, Reynes JM, Hulin A. Acute renal failure associated with dengue fever in French Guiana. Nephron 1999;83:183.

36. Kabra SK, Junepa R, Madhulika, Jain Y, Singhal T, Dar $\mathrm{L}$ et al. Myocardial disfunction in children with dengue haemorrhagic fever. Nat I Med J India 1998;11:59-61.
37. Wali JP, Biswas A, Chandra S, Malhotra A, Aggarwal $\mathbf{P}$, Hamda $\mathbf{R}$ et al. Cardiac involvement in Dengue Haemorrhagic Fever. Int J Cardiol 1998;64:31-6.

38. Khongphatthanayothin A, Suesaowalak M, Muangmingsook S, Bhattarakosol P, Panchaaroen C. Hemodynamic profiles of patients with dengue hemorrhagic fever during toxic stage: an echocardiographic study. Intensive Care Med 2003;29:570-4.

39. Lum LC, Thong MK, Cheah YK, Lam SK. Dengueassociated adult respiratory distress syndrome. Ann Trop Paediatr 1995;15:335-9.

40. Liam CK, Yap BH, Lam SK. Dengue fever complicated by pulmonary haemorrhage manifesting as haemoptysis. J Trop Med Hyg 1993;96:197-200.

41. Ramanathan M, Duraisamy G. Haemophagocytosis in dengue haemorrhagic fever: a case report. Ann Acad Med Singapore 1991;20:803-4.

42. Rueda E, Méndez A, González G. Síndrome hemofagocítico asociado con dengue hemorrágico. Biomédica 2002:22:160-6. 